\title{
The Formula of Kinetic Energy for the Closed Planar Homothetic Direct Motions
}

\author{
Ayhan Tutar, Esra Inan \\ Department of Mathematics, Ondokuz Mayis University, Samsun, Turkey \\ Email: atutar@omu.edu.tr, esra.unsal55@gmail.com
}

Received 23 March 2015; accepted 24 May 2015; published 27 May 2015

Copyright $@ 2015$ by authors and Scientific Research Publishing Inc.

This work is licensed under the Creative Commons Attribution International License (CC BY). http://creativecommons.org/licenses/by/4.0/

cC) (i) Open Access

\begin{abstract}
In this paper, during one-parameter closed planar homothetic direct motions, the formula of kinetic energy is expressed. Then we show the relation between the formula of kinetic energy and the Steiner formula. We investigate some properties of closed planar homothetic motions. These motions appear between two coordinate systems, fixed and moving (direct motion). Finally, we show how the results can be applied to experimentally measured motions. As an example, we consider a motion of winch in the sagittal direction. We obtain the formula of kinetic energy for the motion of winch during one-parameter closed planar homothetic direct motions.
\end{abstract}

\section{Keywords}

Steiner Formula, Kinetic Energy, Planar Kinematics, Homothetic Motions

\section{Introduction}

Jacob Steiner established some properties of the area of a path of a point for a geometrical object rolling on a line and making a complete turn. In planar kinematics, the Steiner formula describes the dependence of the area of a curve, determined by a closed motion of one point, on the position of this point [1]. The Steiner area formula and the Holditch theorem during one-parameter closed planar homothetic motions were expressed by A. Tutar and N. Kuruoglu [2]. During one-parameter closed planar homothetic motions, the expression of the Steiner formula was calculated relative to fixed coordinate system. The points of the fixed plane which enclose the same area lie on a circle or a line in the fixed coordinate system. If it is a circle, then the center of this circle is called the Steiner point $(h=1)$ [3] [4].

Dathe H. and Gezzi R. expressed the formula of kinetic energy for the closed planar kinematics [5]. In our previous paper [6], we introduced a case distinction between movements with or without a vanishing winding number, which was important for practical purposes in mechanics. In this paper, we calculate the formula of ki- 
netic energy for closed planar homothetic direct motions. In particular, we show the relation between the formula of kinetic energy and the Steiner formula.

As an example, Dathe H. and Gezzi R. have chosen the sagittal part of the movement of the human leg during walking for planar kinematics [7]. Since our intention is not simply to recapitulate some classical results, we consider their practical application. We wish to use them in order to characterize experimental data such as the ones related to the motion of winch. For elaboration, we consider the sagittal motion of a winch which is described by a double hinge being fixed and moving as an example. We obtain the formula of kinetic energy for the motion of winch.

\section{The Kinetic Energy in Planar Homothetic Direct Motion}

We consider one parameter closed planar homothetic motion between two reference systems: the fixed $E^{\prime}$ and the moving $E$, with their origins $\left(O, O^{\prime}\right)$ and orientations. Then, we take into account motion relative to the fixed coordinate system (direct motion).

By taking displacement vectors $O O^{\prime}=U$ and $O^{\prime} O=U^{\prime}$, the total angle of rotation $\alpha(t)$, the motion defined by the transformation

$$
X^{\prime}(t)=h(t) R(t) X+U^{\prime}(t)
$$

is called one-parameter closed planar homothetic motion and denoted by $E / E^{\prime}$, where $h$ is a homothetic scale of the motion $E / E^{\prime}, X$ and $X^{\prime}$ are the position vectors with respect to the moving and fixed rectangular coordinate systems of a point $X \in E$, respectively. The homothetic scale $h$ and the vectors $X^{\prime}$ and $U, U^{\prime}$ are continuously differentiable functions of a real parameter $t$.

With the coordinates

$$
X^{\prime}(t)=\left(\begin{array}{l}
x_{1}^{\prime}(t) \\
x_{2}^{\prime}(t)
\end{array}\right), U^{\prime}(t)=\left(\begin{array}{l}
u_{1}^{\prime}(t) \\
u_{2}^{\prime}(t)
\end{array}\right), X=\left(\begin{array}{l}
x_{1} \\
x_{2}
\end{array}\right), U(t)=\left(\begin{array}{l}
u_{1}(t) \\
u_{2}(t)
\end{array}\right)
$$

and rotation matrice

Equation (1) reads components

$$
R(t)=\left(\begin{array}{cc}
\cos (\alpha(t)) & -\sin (\alpha(t)) \\
\sin (\alpha(t)) & \cos (\alpha(t))
\end{array}\right)
$$

$$
\begin{aligned}
& x_{1}^{\prime}(t)=\cos (\alpha(t))\left(h(t) x_{1}-u_{1}\right)-\sin (\alpha(t))\left(h(t) x_{2}-u_{2}\right), \\
& x_{2}^{\prime}(t)=\sin (\alpha(t))\left(h(t) x_{1}-u_{1}\right)+\cos (\alpha(t))\left(h(t) x_{2}-u_{2}\right) .
\end{aligned}
$$

From Equation (3), by differentiation with respect to $t$, we have

$$
\begin{aligned}
& \dot{x}_{1}^{\prime}=-\left(h x_{1}-u_{1}\right) \sin \alpha \cdot \dot{\alpha}+\left(\dot{h} x_{1}-\dot{u}_{1}\right) \cos \alpha-\left(h x_{2}-u_{2}\right) \cos \alpha \cdot \dot{\alpha}-\left(\dot{h} x_{2}-\dot{u}_{2}\right) \sin \alpha, \\
& \dot{x}_{2}^{\prime}=\left(h x_{1}-u_{1}\right) \cos \alpha \cdot \dot{\alpha}+\left(\dot{h} x_{1}-\dot{u}_{1}\right) \sin \alpha-\left(h x_{2}-u_{2}\right) \sin \alpha \cdot \dot{\alpha}+\left(\dot{h} x_{2}-\dot{u}_{2}\right) \cos \alpha .
\end{aligned}
$$

A moment with a first order in the time derivatives can be introduced by

$$
S=\frac{1}{2} \int\left[\left(\dot{x}_{1}^{\prime}\right)^{2}+\left(\dot{x}_{1}^{\prime}\right)^{2}\right] \mathrm{d} t
$$

which is the integral over the kinetic energy of a point with mass $M=1$.

So, we can calculate this equation using Equation (4)

$$
\begin{aligned}
\left(\dot{x}_{1}^{\prime}\right)^{2}+\left(\dot{x}_{1}^{\prime}\right)^{2}= & h^{2}\left(x_{1}^{2}+x_{2}^{2}\right) \dot{\alpha}^{2}+\left(x_{1}^{2}+x_{2}^{2}\right) \dot{h}^{2}+\left(u_{1}^{2}+u_{2}^{2}\right) \dot{\alpha}^{2}+\dot{u}_{1}^{2}+\dot{u}_{2}^{2}+2 u_{1} \dot{u}_{2} \dot{\alpha}-2 u_{2} \dot{u}_{1} \dot{\alpha} \\
& +x_{1}\left(-2 h u_{1} \dot{\alpha}^{2}-2 \dot{h} \dot{u}_{1}-2 h \dot{u}_{2} \dot{\alpha}+2 h u_{2} \dot{\alpha}\right)+x_{2}\left(-2 h u_{2} \dot{\alpha}^{2}-2 \dot{h} \dot{u}_{2}+2 h \dot{u}_{1} \dot{\alpha}-2 h u_{1} \dot{\alpha}\right) .
\end{aligned}
$$

If Equation (6) is replaced in Equation (5),

$$
\begin{aligned}
2 S= & \left(x_{1}^{2}+x_{2}^{2}\right) \int\left(h^{2} \dot{\alpha}^{2}+\dot{h}^{2}\right) \mathrm{d} t+\int\left[\left(u_{1}^{2}+u_{2}^{2}\right) \dot{\alpha}^{2}+\dot{u}_{1}^{2}+\dot{u}_{2}^{2}+2 u_{1} \dot{u}_{2} \dot{\alpha}-2 u_{2} \dot{u}_{1} \dot{\alpha}\right] \mathrm{d} t \\
& +x_{1} \int\left[-2 h u_{1} \dot{\alpha}^{2}-2 \dot{h} \dot{u}_{1}-2 h \dot{u}_{2} \dot{\alpha}+2 \dot{h} u_{2} \dot{\alpha}\right] \mathrm{d} t+x_{2} \int\left[-2 h u_{2} \dot{\alpha}^{2}-2 \dot{h} \dot{u}_{2}+2 h \dot{u}_{1} \dot{\alpha}-2 \dot{h} u_{1} \dot{\alpha}\right] \mathrm{d} t
\end{aligned}
$$


is found.

If $X=0\left(x_{1}=0, x_{2}=0\right)$ is taken, then, for the formula of kinetic energy of the origin point we have

$$
2 S_{O}=\int\left[\left(u_{1}^{2}+u_{2}^{2}\right) \dot{\alpha}^{2}+\dot{u}_{1}^{2}+\dot{u}_{2}^{2}+2 u_{1} \dot{u}_{2} \dot{\alpha}-2 u_{2} \dot{u}_{1} \dot{\alpha}\right] \mathrm{d} t .
$$

If Equation (8) is replaced in Equation (7),

$$
\begin{aligned}
2\left(S-S_{o}\right)= & \left(x_{1}^{2}+x_{2}^{2}\right) \int\left(h^{2} \dot{\alpha}^{2}+\dot{h}^{2}\right) \mathrm{d} t+x_{1} \int\left[-2 h u_{1} \dot{\alpha}^{2}-2 \dot{h}_{1}-2 h \dot{u}_{2} \dot{\alpha}+2 \dot{h} u_{2} \dot{\alpha}\right] \mathrm{d} t \\
& +x_{2} \int\left[-2 h u_{2} \dot{\alpha}^{2}-2 \dot{h} \dot{u}_{2}+2 h \dot{u}_{1} \dot{\alpha}-2 \dot{h} u_{1} \dot{\alpha}\right] \mathrm{d} t
\end{aligned}
$$

can be written.

Now we consider the case in which the motion is closed and naturally parametrized. Other cases will be discussed in an another publication. Then, it follows $\mathrm{d} t \rightarrow \mathrm{d} \alpha, \dot{\alpha} \rightarrow \alpha^{\prime}=\frac{\mathrm{d} \alpha}{\mathrm{d} \alpha}=1$ and $\int \rightarrow \oint$. With those assumptions, we obtain

$$
\begin{aligned}
2\left(S-S_{O}\right)= & x_{1} \oint\left[-2 h u_{1}-2 \mathrm{~d} h \mathrm{~d} u_{1}-2 h \mathrm{~d} u_{2}+2 \mathrm{~d} h u_{2}\right] \mathrm{d} \alpha \\
& +x_{2} \oint\left[-2 h u_{2}-2 \mathrm{~d} h \mathrm{~d} u_{2}+2 h \mathrm{~d} u_{1}-2 \mathrm{~d} h u_{1}\right] \mathrm{d} \alpha \\
& +\left(x_{1}^{2}+x_{2}^{2}\right) \oint\left(h^{2}+(\mathrm{d} h)^{2}\right) \mathrm{d} \alpha .
\end{aligned}
$$

Equation (13) of [6]: $U=\left(\begin{array}{l}u_{1} \\ u_{2}\end{array}\right)$,

$$
\begin{aligned}
& u_{1}=h p_{1}+p_{2} \frac{\mathrm{d} h}{\mathrm{~d} \alpha}-\frac{\mathrm{d} u_{2}}{\mathrm{~d} \alpha}, \\
& u_{2}=h p_{2}-p_{1} \frac{\mathrm{d} h}{\mathrm{~d} \alpha}-\frac{\mathrm{d} u_{1}}{\mathrm{~d} \alpha} .
\end{aligned}
$$

If Equation (13) of [6] is respectively replaced at coefficients of $x_{1}$ and $x_{2}$ in Equation (10) and by calculating necessary operations,

$$
\begin{aligned}
2\left(S-S_{O}\right)= & x_{1} \oint\left[-2 h^{2} p_{1} \mathrm{~d} \alpha-2 h p_{2} \mathrm{~d} h-2 \frac{\mathrm{d} h}{\mathrm{~d} \alpha} \mathrm{d} u_{1}+2 \mathrm{~d} h u_{2}\right] \\
& +x_{2} \oint\left[-2 h^{2} p_{2} \mathrm{~d} \alpha+2 h p_{1} \mathrm{~d} h-2 \frac{\mathrm{d} h}{\mathrm{~d} \alpha} \mathrm{d} u_{2}-2 \mathrm{~d} h u_{1}\right] \\
& +\left(x_{1}^{2}+x_{2}^{2}\right) \oint h^{2} \mathrm{~d} \alpha+\left(x_{1}^{2}+x_{2}^{2}\right) \oint \frac{(\mathrm{d} h)^{2}}{\mathrm{~d} \alpha}
\end{aligned}
$$

is found.

We consider Equations (10), (14), (15) and (18) of [6], namely,

$$
\begin{gathered}
m=\oint h^{2} \mathrm{~d} \alpha=h^{2}\left(t_{0}\right) \oint \mathrm{d} \alpha=h^{2}\left(t_{0}\right) 2 \pi v, \\
a^{*}=\oint \underbrace{\left(-2 h^{2} p_{1} \mathrm{~d} \alpha\right)}_{a}+\underbrace{\oint\left(-2 h \mathrm{~d} h p_{2}+h \mathrm{~d} u_{2}+u_{2} \mathrm{~d} h\right)}_{\mu_{1}}, \\
b^{*}=\underbrace{\left(-2 h^{2} p_{2} \mathrm{~d} \alpha\right)}_{b}+\underbrace{\oint\left(2 h \mathrm{~d} h p_{1}-h \mathrm{~d} u_{1}-u_{1} \mathrm{~d} h\right)}_{\mu_{2}},
\end{gathered}
$$

and

$$
2\left(F-F_{o}\right)=\left(x_{1}^{2}+x_{2}^{2}\right) m+a x_{1}+b x_{2}+\mu_{1} x_{1}+\mu_{2} x_{2} .
$$

Finally, if Equations (10), (14), (15) and (18) of [6] are replaced in Equation (11), 


$$
\begin{aligned}
2\left(S-S_{O}\right)= & 2\left(F-F_{O}\right)+\left(x_{1}^{2}+x_{2}^{2}\right) \oint \frac{(\mathrm{d} h)^{2}}{\mathrm{~d} \alpha}+x_{1} \oint\left[\mathrm{d} h u_{2}-h \mathrm{~d} u_{2}-2 \frac{\mathrm{d} h}{\mathrm{~d} \alpha} \mathrm{d} u_{1}\right] \\
& +x_{2} \oint\left[-\mathrm{d} h u_{1}+h \mathrm{~d} u_{1}-2 \frac{\mathrm{d} h}{\mathrm{~d} \alpha} \mathrm{d} u_{2}\right]
\end{aligned}
$$

is arrived at the relation between the formula of kinetic energy and the formula for the area.

\section{Example 1. The Direct Motion of Winch}

The motion of winch has a double hinge and "a double hinge" is mean that it has two systems, a fixed arm and a moving arm of winch (Figure 1). There is a control panel of winch at the origin of moving system. " $L$ " arm can extend or retract by $h$ parameter. Also we define using the condition $m=0$ (Equation (10) of [6]), the Steiner line and the total angle in relation to the double hinge. So we must use it for this section.

By considering Equation (36) of [6],

$$
\begin{aligned}
& x_{1}^{\prime}(t)=\cos (l(t)-k(t))\left(h(t) x_{1}+L \cos (k)\right)-\sin (l(t)-k(t))\left(h(t) x_{2}+L \sin (k)\right) \\
& x_{2}^{\prime}(t)=\sin (l(t)-k(t))\left(h(t) x_{1}+L \cos (k)\right)+\cos (l(t)-k(t))\left(h(t) x_{2}+L \sin (k)\right)
\end{aligned}
$$

and if we calculate the time derivative of this,

$$
\begin{aligned}
\dot{x}_{1}^{\prime}= & -\left(h x_{1}+L \cos k\right) \sin (l-k)(\dot{l}-\dot{k})+\left(\dot{h} x_{1}-L \sin k \cdot \dot{k}\right) \cos (l-k) \\
& -\left(h x_{2}+L \sin k\right) \cos (l-k)(\dot{l}-\dot{k})-\left(\dot{h} x_{2}+L \cos k \cdot \dot{k}\right) \sin (l-k) \\
\dot{x}_{2}^{\prime}= & \left(h x_{1}+L \cos k\right) \cos (l-k)(\dot{l}-\dot{k})+\left(\dot{h} x_{1}-L \sin k \cdot \dot{k}\right) \sin (l-k) \\
& -\left(h x_{2}+L \sin k\right) \sin (l-k)(i-\dot{k})+\left(\dot{h} x_{2}+L \cos k \cdot \dot{k}\right) \cos (l-k)
\end{aligned}
$$

are found.

We must calculate $x_{1}^{2}+x_{2}^{2}$ for the formula of kinetic energy in Equation (5)

$$
\begin{aligned}
2 S= & x_{1} \int\left[2 h L \cos k(i-\dot{k})^{2}-2 \dot{h} L \sin k \cdot \dot{k}-2 h L \cos k \cdot \dot{k}(i-\dot{k})+2 \dot{h} L \sin k(i-\dot{k})\right] \mathrm{d} t \\
& +x_{2} \int\left[2 h L \sin k(\dot{l}-\dot{k})^{2}+2 \dot{h} L \cos k \cdot \dot{k}+2 h L \sin k \cdot \dot{k}(i-\dot{k})+2 \dot{h} L \cos k(\dot{l}-\dot{k})\right] \mathrm{d} t \\
& +\left(x_{1}^{2}+x_{2}^{2}\right) \int h^{2}(\dot{l}-\dot{k})^{2} \mathrm{~d} t+\left(x_{1}^{2}+x_{2}^{2}\right) \int \dot{h}^{2}(\dot{l}-\dot{k})^{2} \mathrm{~d} t+L^{2} \int \dot{l}^{2} \mathrm{~d} t
\end{aligned}
$$

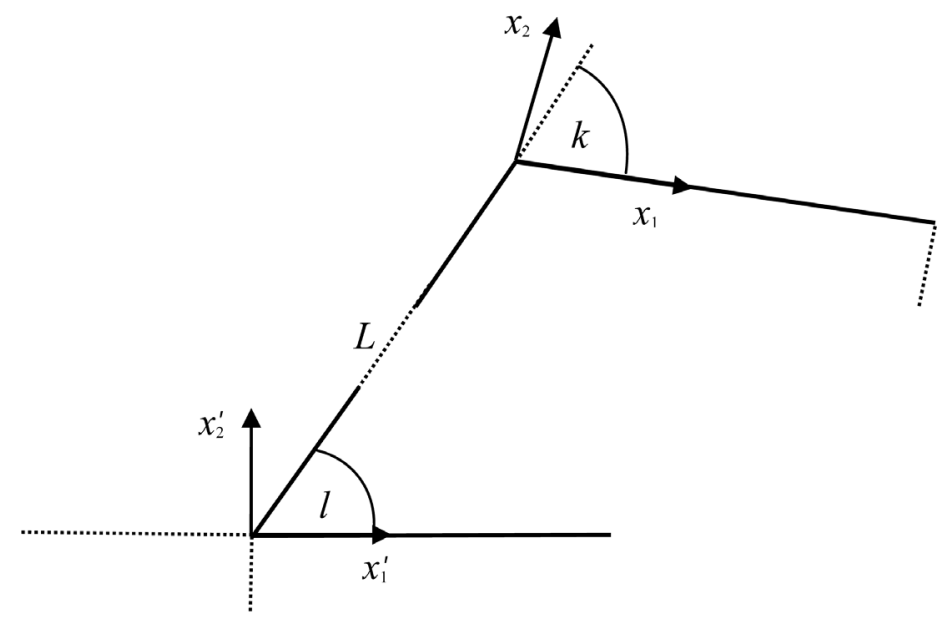

Figure 1. The arms of winch as a double hinge. 
Also we use Equation (35) of [6]

$$
U^{\prime}(t)=\left(\begin{array}{l}
u_{1}^{\prime}(t) \\
u_{2}^{\prime}(t)
\end{array}\right)=\left(\begin{array}{c}
L \cos (l(t)) \\
L \sin (l(t))
\end{array}\right) .
$$

If we calculate the time derivative of this,

$$
\begin{aligned}
& \mathrm{d} u_{1}^{\prime}=-L \sin l \cdot i \mathrm{~d} t, \\
& \mathrm{~d} u_{2}^{\prime}=L \cos l \cdot i \mathrm{~d} t
\end{aligned}
$$

are found. Then if Equation (35) of [6] and Equation (15) are replaced in calculating data $x_{1}^{2}+x_{2}^{2}$ and in Section 2, by using the parameter $\mathrm{d} t \rightarrow \mathrm{d} \alpha, \dot{\alpha} \rightarrow \alpha^{\prime}=\frac{\mathrm{d} \alpha}{\mathrm{d} \alpha}=1$ and $\int \rightarrow \oint$ :

$$
\begin{aligned}
2 S= & \left(x_{1}^{2}+x_{2}^{2}\right) \int_{t_{1}}^{t_{2}} \frac{\mathrm{d} h^{2}}{\mathrm{~d} \alpha}+L^{2} \int_{t_{1}}^{t_{2}} \frac{\mathrm{d} l^{2}}{\mathrm{~d} \alpha}+x_{1} \int_{t_{1}}^{t_{2}}\left[-2 h u_{1} \mathrm{~d} \alpha-2 \frac{\mathrm{d} h}{\mathrm{~d} \alpha} \mathrm{d} u_{1}-2 h \mathrm{~d} u_{2}+2 u_{2} \mathrm{~d} h\right] \\
& +x_{2} \int_{t_{1}}^{t_{2}}\left[-2 h u_{2} \mathrm{~d} \alpha-2 \frac{\mathrm{d} h}{\mathrm{~d} \alpha} \mathrm{d} u_{2}+2 h \mathrm{~d} u_{1}-2 u_{1} \mathrm{~d} h\right] .
\end{aligned}
$$

If Equation (13) of [6] is replaced in Equation (17),

$$
\begin{aligned}
2 S= & \left(x_{1}^{2}+x_{2}^{2}\right) \int_{t_{1}}^{t_{2}} \frac{\mathrm{d} h^{2}}{\mathrm{~d} \alpha}+L^{2} \int_{t_{1}}^{t_{2}} \frac{\mathrm{d} l^{2}}{\mathrm{~d} \alpha}+x_{1} \int_{t_{1}}^{t_{2}}-2 h^{2} p_{1} \mathrm{~d} \alpha+x_{2} \int_{t_{1}}^{t_{2}}-2 h^{2} p_{2} \mathrm{~d} \alpha \\
& +x_{1} \int_{t_{1}}^{t_{2}}\left[-2 h p_{2} \mathrm{~d} h-2 \frac{\mathrm{d} h}{\mathrm{~d} \alpha} \mathrm{d} u_{1}+2 u_{2} \mathrm{~d} h\right]+x_{2} \int_{t_{1}}^{t_{2}}\left[2 h p_{1} \mathrm{~d} h-2 \frac{\mathrm{d} h}{\mathrm{~d} \alpha} \mathrm{d} u_{2}-2 u_{1} \mathrm{~d} h\right] .
\end{aligned}
$$

is found.

Now we can construct Equation (18) as the formula of area.

$$
\begin{aligned}
2 S= & x_{1} \int_{t_{1}}^{t_{2}}-2 h^{2} p_{1} \mathrm{~d} \alpha+x_{2} \int_{t_{1}}^{t_{2}}-2 h^{2} p_{2} \mathrm{~d} \alpha+x_{1} \int_{t_{1}}^{t_{2}}\left[-2 h p_{2} \mathrm{~d} h+h \mathrm{~d} u_{2}+u_{2} \mathrm{~d} h\right] \\
& +x_{2} \int_{t_{1}}^{t_{2}}\left[2 h p_{1} \mathrm{~d} h-h \mathrm{~d} u_{1}-u_{1} \mathrm{~d} h\right]+\left(x_{1}^{2}+x_{2}^{2}\right) \int_{t_{1}}^{t_{2}} \frac{\mathrm{d} h^{2}}{\mathrm{~d} \alpha}+L^{2} \int_{t_{1}}^{t_{2}} \frac{\mathrm{d} l^{2}}{\mathrm{~d} \alpha} \\
& +x_{1} \int_{t_{1}}^{t_{2}}\left[u_{2} \mathrm{~d} h-h \mathrm{~d} u_{2}-2 \frac{\mathrm{d} h}{\mathrm{~d} \alpha} \mathrm{d} u_{1}\right]+x_{2} \int_{t_{1}}^{t_{2}}\left[-u_{1} \mathrm{~d} h+h \mathrm{~d} u_{1}-2 \frac{\mathrm{d} h}{\mathrm{~d} \alpha} \mathrm{d} u_{2}\right] .
\end{aligned}
$$

We consider Equations (40), (41) of [6], namely,

$$
\begin{gathered}
\oint \underbrace{\left(-2 h^{2} p_{1} \mathrm{~d} \alpha\right)}_{a}+\underbrace{\oint\left(-2 h \mathrm{~d} h p_{2}+h \mathrm{~d} u_{2}+u_{2} \mathrm{~d} h\right)}_{\mu_{1}}=a^{*}, \\
\underbrace{\left(-2 h^{2} p_{2} \mathrm{~d} \alpha\right)}_{b}+\underbrace{\oint\left(2 h \mathrm{~d} h p_{1}-h \mathrm{~d} u_{1}-u_{1} \mathrm{~d} h\right)}_{\mu_{2}}=b^{*}, \\
2 F=\left(a+\mu_{1}\right) x_{1}+\left(b+\mu_{2}\right) x_{2}
\end{gathered}
$$

If Equations (40) and (41) of [6] are replaced in Equation (19),

$$
\begin{aligned}
2 S= & 2 F+\left(x_{1}^{2}+x_{2}^{2}\right) \int_{t_{1}}^{t_{2}} \frac{\mathrm{d} h^{2}}{\mathrm{~d} \alpha}+L^{2} \int_{t_{1}}^{t_{2}} \frac{\mathrm{d} l^{2}}{\mathrm{~d} \alpha}+x_{1} \int_{t_{1}}^{t_{2}}\left[u_{2} \mathrm{~d} h-h \mathrm{~d} u_{2}-2 \frac{\mathrm{d} h}{\mathrm{~d} \alpha} \mathrm{d} u_{1}\right] \\
& +x_{2} \int_{t_{1}}^{t_{2}}\left[-u_{1} \mathrm{~d} h+h \mathrm{~d} u_{1}-2 \frac{\mathrm{d} h}{\mathrm{~d} \alpha} \mathrm{d} u_{2}\right]
\end{aligned}
$$

is arrived at the relation between the formula of kinetic energy and the area formula for application. 


\section{References}

[1] Steiner, J. (1840) Von dem Krümmungs-Schwerpuncte ebener Curven. Journal für die Reine und Angewandte Mathematik, 21, 33-63. http://dx.doi.org/10.1515/crll.1840.21.33

[2] Tutar, A. and Kuruoğlu, N. (1999) The Steiner Formula and the Holditch Theorem for the Homothetic Motions on the Planar Kinematics. Mechanism and Machine Theory, 34, 1-6. http://dx.doi.org/10.1016/S0094-114X(98)00028-7

[3] Müller, H.R. (1978) Verallgemeinerung einer Formel von Steiner. Abhandlungen der Braunschweigischen Wissenschaftlichen Gesellschaft, 29, 107-113.

[4] Müller, H.R. (1978) Über Trägheitsmomente bei Steinerscher Massenbelegung. Abhandlungen der Braunschweigischen Wissenschaftlichen Gesellschaft, 29, 115-119.

[5] Dathe, H. and Gezzi, R. (2014) Addenda and Erratum to: Characteristic Directions of Closed Planar Motions. Zeitschrift für Angewandte Mathematik und Mechanik, 94, 551-554. http://dx.doi.org/10.1002/zamm.201300230

[6] Inan, E. and Tutar, A. (2014) Characteristic Directions of Closed Planar Homothetic Direct Motions. Submitted.

[7] Dathe, H. and Gezzi, R. (2012) Characteristic Directions of Closed Planar Motions. Zeitschrift für Angewandte Mathematik und Mechanik, 92, 2-13. http://dx.doi.org/10.1002/zamm.201100178 